\title{
Effects of mental fatigue on psychophysiological, cognitive responses, and technical skills in small-sided soccer games in amateur players
}

Authors' Contribution: A Study Design

B Data Collection

C Statistical Analysis

D Data Interpretation

E Manuscript Preparation

F Literature Search

G Funds Collection

\author{
Yusuf Soylu ABEF, Ersan Arslan ACDE
}

Faculty of Sport Sciences, Tokat Gaziosmanpasa University, Tokat, Turkey

\section{abstract}

Background:

Material and methods:

The purpose of this study was to assess the effects of mental fatigue (MF) on the psychophysiological and cognitive responses and technical skills in small-sided games (SSGs) in amateur soccer players.

18 soccer players played 2vs2, 3vs3, and 4vs4 SSGs under 2 different playing conditions: MF+SSGs and SSGs. The Feeling Scale (FS), Felt Arousal Scale (FAS), Visual Analogue Scale-Anxiety (VAS-A) and Motivation (MTV) were determined after each SSGs bout. A Trail-Making Test (TMT) was also determined in the beginning and at the end of each SSGs. Technical performance was continuously recorded with a camera during all SSGs.

Results: The results demonstrated that MF induced higher negative psychophysiological and cognitive responses ( $p \leq 0.05, d=$ ranging from 0.73 to 2.78 ) in 2 vs2 and 3vs3 SSGs compared to 4vs4 SSGs except for VAS-A and MTV responses. Furthermore, the MF intervention also induced an adverse effect on technical skills ( $p \leq 0.05, d=$ ranging from 0.65 to 2.50 ) in 2 vs2 and $3 v s 3$ SSGs compared to $4 v s 4$ SSGs except for interception, tackle and two touches skills.

Conclusions: These findings showed that MF impacted the psychophysiological and cognitive responses, and technical skills during SSGs. Coaches should take MF into account before planning SSGs.

Key words: soccer, mental fatigue, technical performances, psychological responses.

\section{article details}

Article statistics: Word count: 1,913; Tables: 3; Figures: 1 References: 46

Received: January 2021; Accepted: June 2021; Published: December 2021

Full-text PDF: http://www.balticsportscience.com

Copyright @ Gdansk University of Physical Education and Sport, Poland

Indexation: Celdes, Clarivate Analytics Emerging Sources Citation Index (ESCl), CNKI Scholar (China National Knowledge Infrastructure), CNPIEC, DOAJ, EBSCO - Central \& Eastern European Academic Source, EBSCO - SPORTDiscus, EBSCO Discovery Service, Google Scholar, Index Copernicus, J-Gate, Naviga (Softweco, Primo Central (ExLibris), ProQuest - Family Health, ProQuest - Health \& Medical Complete, ProQuest - Illustrata: Health Sciences, ProQuest Nursing \& Allied Health Source, Summon (Serials Solutions/ProQuest, TDOne (TDNet), Ulrich's Periodicals Directory/ ulrichsweb, WorldCat (OCLC)

Funding: This research received no specific grant from any funding agency in the public, commercial, or not-for-profit sectors.

Conflict of interests: Corresponding author:

Open Access License:
Authors have declared that no competing interest exists.

Yusuf Soylu, Faculty of Sport Sciences, Tasliciftlik Campus, Tokat Gaziosmanpasa University, 60250 Tokat, Turkey; Telephone: +90 (356) 25216 16; e-mail: Oyusufsoylu@gmail.com

This is an open access article distributed under the terms of the Creative Commons Attribution-Non-Commercial-NoDerivatives 4.0 International (https://creativecommons.org/licenses/by-nc-nd/4.0/), which permits use, distribution, and reproduction in any medium, provided the original work is properly cited, the use is non-commercial and is otherwise in compliance with the license. 


\section{INTRODUCTION}

The soccer game concept, which includes aerobic capacity, feelings and perceptual-cognitive skills, is related to physical, technical-tactical, psychological and cognitive demands [1-3]. Therefore, many types of soccer training sessions are mainly used to develop different strategies in the face of increasing and changing in-game contexts. Small-sided games (SSGs), one of the commonly used time-efficient training methods, a re an effective role in order to improve soccer-specific aerobic capacity, technical skills, and psychological conditions transferred from training activities to a match $[4,5]$.

Recent studies have focused on psychological and mental factors in SSGs including mood, enjoyment, and decision making in soccer players [6-8]. In the light of these studies, the players are not only exposed to the external loading, but also feeling higher internal loading during the SSGs. Several alternative training methods have been used to enhance the performance and technical capabilities of young soccer players [5, 9]. In contrast to traditional training drills, the SSGs simulate soccer game conditions with an enjoyable, effective and time-efficient training approach [3, 4,10$]$. Generally, the characteristics of SSGs training have been adapted to the integration of physical development, team cohesion, technical and tactical variability to soccer matches $[11,12]$.

Most of the previous studies have shown that performance in SSGs is measured by different methods such as internal and external loading [3, 5, 13, 14]. However, a popular topic on soccer in recent studies has focused on some interventions such as mental fatigue [15-17] that can affect players' performance in SSGs. Mental fatigue (MF) refers to a psychobiological state representing a feeling of tiredness and lack of energy, decreased motivation and alertness by leading to long periods of cognitive activity [18-20]. Researchers state that MF has been linked to increasing time on a task which changes cognitive and behavioural performance [21, 22].

Soccer-specific psychophysiological [17] and cognitive performance [16], and technical skills [23] are negatively affected by MF. Considering that SSGs are an important part of game-based training in soccer, MF interventions might play a key role in decreasing soccer game demands. Therefore, we hypothesise that MF would negatively affect the psychophysiological, cognitive and technical performance responses, such as feelings, response attention, and technical skills especially interception, successful and unsuccessful tackle during SSGs.

\section{MATERIAL AND METHODS}

\section{PARTICIPANTS}

18 male soccer players (age: $19.1 \pm 1.2$ years, body height $176.8 \pm 6.2 \mathrm{~cm}$ ) participated in this study. All the players were members of an amateur soccer team, competing in the regional league. They were familiar with a training workload of $>4$ training units per week, each lasting 90-minutes and a league match for more than 5 years. Before signing the informed consent form, detailed information was declared to the players. The study protocol was approved by Tokat Gaziosmanpasa University the Research Ethics Committee (2021/E33490967-044-22125) and was conducted with the institutional ethical requirements for human experimentation in accordance with the Declaration of Helsinki. 
Table 1. The dimensions of SSGs

\begin{tabular}{cccccc} 
& & \multicolumn{2}{c}{ Work : Rest } & \multicolumn{2}{c}{ Pitch Dimensions } \\
\cline { 3 - 6 } SSGs Formats & Bouts & $\begin{array}{c}\text { Bouts } \\
(\mathrm{min})\end{array}$ & $\begin{array}{c}\text { Resting } \\
(\mathrm{min})\end{array}$ & $\begin{array}{c}\text { Pitch } \\
\text { Size }(\mathrm{m})\end{array}$ & $\begin{array}{c}\text { Area } \\
\text { Per Player }\left(\mathrm{m}^{2}\right)\end{array}$ \\
\hline 2v2+MG & 2 & & $15 \times 27$ & 100 \\
3 V 3+MG & 4 & 3 & 2 & $20 \times 30$ & \\
4 V $4+M G$ & 4 & & $25 \times 32$ & \\
\hline
\end{tabular}

\section{INSTRUMENTS}

Feeling Scale. The Feeling Scale (FS) is a psychophysiological bipolar scale of 11 items and ranging from +5 to -5 . The verbal anchor asked is "How do you feel right now?" [24].

Felt Arousal Scale. The Felt Arousal Scale (FAS) is a psychophysiological measure which assesses pleasure and displeasure. Players were asked to report on "How aroused do you actually feel?". The FAS is a single item bi-polar scale ranging from low arousal (1) to high arousal (6) [25].

Visual Analogue Scale-Anxiety. The Visual Analogue Scale-Anxiety (VAS-A) is a self-report measure which assesses anxiety. The VAS-A used consisted of a $100 \mathrm{~mm}$ horizontal line labelled with 'not at all anxious' at one end (score of 0 ) and 'extremely anxious' at the other (score of 100) [26].

Motivation. The Motivation (MTV) measure time trial was measured using a single item ("I am motivated to do the time trial") scored on a 5-point Likert scale $(0=$ not at all, $1=$ a little bit, $2=$ somewhat, 3 =very much, $4=$ extremely) [27].

Trail-Making Test. The Trail-Making Test (TMT) is a neuropsychological test measure of attention, speed, and cognitive flexibility. The TMT includes two different paper/pencil parts a TMT-A and TMT-B. Participants performed each part as fast as possible. The TMT is widely used as a reliable and valid measure of scanning, visuomotor tracking, split attention and cognitive flexibility [28].

\section{TECHNICAL ACTIONS}

All SSGs were recorded using a high-definition camera (SONY HDR-CX130, Sony Corp., Tokyo, Japan), and the analysis of the technical actions of each SSGs was performed using eAnalyse soccer match-analysis software (Espor Digital, Ankara, Turkey). The technical variables determined from video recordings were interception, successful and unsuccessful tackle, 1 Touch (Succ-Unsucc), 2 Touches (Succ-Unsucc), and a lost ball.

\section{EXPERIMENTAL PROTOCOL}

A randomized counterbalanced cross-over design was used to compare MF on the psychophysiological, cognitive responses and technical skills in SSGs in amateur players. All players were randomly separated into two groups as MF with SSGs (MF+SSGs) and SSGs without MF (SSGs). The MF intervention protocol was completed for 17 days every 48 hours to prevent any risk of physical, psychological and mental state. A session familiarizing with the tests was carried out a week before training days. All SSGs sessions used a minigoal, and were completed at the same time of day and on the same pitch.

\section{INTERVENTIONS}

Mental Fatigue Intervention. The paper version of the 30-min Stroop task was performed to induce MF. Previous studies $[8,16]$ demonstrated that the Stroop task is used to induce MF 
in soccer. Before the MF intervention, all players were provided with clarification by the practitioner. The Stroop task, consisting of four words (red, blue, green and yellow), was shown in a random order, following by a $1.5 \mathrm{~s}$ resting interval. Players were also instructed to quickly and accurately finish the task in competition with the other teammates. This prolonged cognitive task requires intensive attention and automatic response inhibition [29].

SSGs Intervention. A standard 15-minutes warm-up protocol consists of jogging, dynamic stretching, and soccer-specific actions that were performed by players. The SSGs protocol is formed by two conditions (MF+SSGs and SSGs) and three different games (2v2, 3v3 and $4 \mathrm{v} 4$ ), as detailed in Table 1. All SSGs sessions were performed with 48 hours of scheduled rest. Before the use, VAS-A, FS, FAS, MTV and TMT were explained to the players, and then VAS-A, FS, MTV and TMT were used before the sessions. During the bouts of SSGs, VAS-A, FS, FAS, MTV and technical actions were used. All assessments were made post sessions in all SSGs (Figure 1).

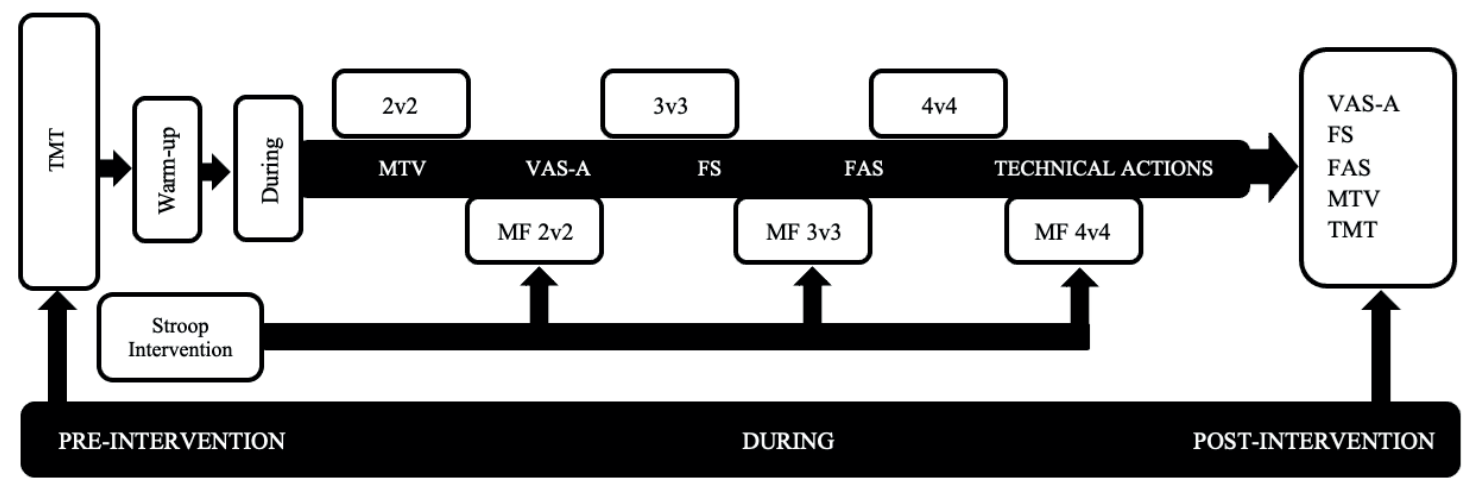

Fig. 1. Study design

\section{STATISTICAL ANALYSES}

All the data was collected pre, during and post all SSGs and to analyse each dependent variable using a paired-sample t-test to compare MF+SSGs and SSGs sessions and present means with standard deviation (SD), mean differences, means with a 95\% confidence interval (95\% CI). To estimate differences between pre and post MF effects, Cohens $d$ and rating (effect size) were calculated for each dependent variable. The thresholds for effect size statistics were as follows: 0.2 - trivial; 0.6 - small; 1.2 - moderate; 2.0 - large; and $<2.0$ - very large [30]. Statistical analyses were performed with SPSS software version 24.0 (SPSS, Version 24.0 for Windows; SPSS Inc., Chicago, IL, United States). The level of statistical significance was set at $\mathrm{p} \leq 0.05$.

\section{RESULTS}

Table 1 presents comparisons of psychophysiological differences between MF+SSGs and SSGs 2v2, 3v3, and 4v4 formats. The results demonstrated that MF induced higher negative psychophysiological and cognitive responses ( $\mathrm{p} \leq 0.05, \mathrm{~d}=$ ranging from 0.73 to 2.78 ) in $2 \mathrm{vs} 2$ and 3vs3 SSGs compared to 4vs4 SSGs except for VAS-A and MTV responses. 
Table 2. Psychophysiological and cognitive responses of amateur soccer players to SSGs+MF and SSGs conditions

\begin{tabular}{|c|c|c|c|c|c|c|c|}
\hline & & Variables & $\begin{array}{c}\text { MF+SSGs } \\
\text { Mean } \pm \text { SD }\end{array}$ & $\begin{array}{c}\text { SSGs } \\
\text { Mean } \pm \text { SD }\end{array}$ & $\begin{array}{c}\text { Mean } \\
\text { Difference }\end{array}$ & $\% 95 \mathrm{Cl}$ & $\begin{array}{l}\text { Cohens d } \\
\text { and Rating }\end{array}$ \\
\hline \multirow{18}{*}{ 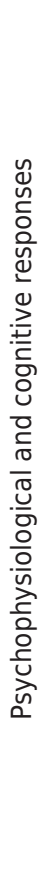 } & \multirow{6}{*}{$\begin{array}{l}\frac{0}{0} \\
\frac{0}{n} \\
\frac{1}{0} \\
\stackrel{1}{N}\end{array}$} & VAS-A & $2.56 \pm 2.75^{*}$ & $1.00 \pm 0.59$ & 1.56 & $-2.91--0.20$ & 0.78; Moderate \\
\hline & & FS & $0.61 \pm 1.33^{*}$ & $3.11 \pm 3.22$ & -2.5 & $0.88-4.12$ & -1.01; Moderate \\
\hline & & FAS & $2.28 \pm 0.67 *$ & $3.72 \pm 1.27$ & -1.44 & $-2.15--0.74$ & -1.42; Large \\
\hline & & MTV & $1.50 \pm 0.51 *$ & $3.28 \pm 0.75$ & -1.78 & $-2.28--1.28$ & -2.78; Very Large \\
\hline & & TMT-1 & $17.24 \pm 2.62 *$ & $13.20 \pm 4.53$ & 4.04 & $1.57-6.51$ & 1.10; Moderate \\
\hline & & TMT-2 & $24.46 \pm 5.10 *$ & $20.23 \pm 4.46$ & 4.23 & $0.72-7.73$ & 0.88; Moderate \\
\hline & \multirow{6}{*}{$\begin{array}{l}\frac{0}{0} \\
\frac{0}{n} \\
\frac{1}{0} \\
\stackrel{1}{m}\end{array}$} & VAS-A & $2.61 \pm 3.16^{*}$ & $0.89 \pm 1.08$ & 1.72 & $-3.12--0.33$ & 0.73; Moderate \\
\hline & & FS & $1.22 \pm 0.88^{*}$ & $3.67 \pm 3.16$ & -2.45 & $0.91-3.98$ & -1.06; Moderate \\
\hline & & FAS & $2.17 \pm 1.04^{*}$ & $3.78 \pm 1.17$ & -1.61 & $-2.32--0.90$ & -1.45; Large \\
\hline & & MTV & $2.00 \pm 0.77^{*}$ & $3.22 \pm 0.88$ & -1.22 & $-1.78--0.67$ & -1.48; Large \\
\hline & & TMT-1 & $17.23 \pm 1.31^{*}$ & $11.96 \pm 4.22$ & 5.27 & $0.22-3.50$ & 1.69; Large \\
\hline & & TMT-2 & $19.66 \pm 2.93 *$ & $15.52 \pm 6.10$ & 4.14 & $1.30-7.00$ & 0.87 ; Moderate \\
\hline & \multirow{6}{*}{$\begin{array}{l}\frac{0}{0} \\
\frac{0}{1} \\
\frac{1}{0} \\
\dot{d}\end{array}$} & VAS-A & $2.22 \pm 2.94$ & $0.61 \pm 0.50$ & 1.61 & $-2.99--0.23$ & - \\
\hline & & FS & $1.94 \pm 0.54 *$ & $3.94 \pm 3.46$ & -2.0 & $0.26-3.74$ & -0.81 ; Moderate \\
\hline & & FAS & $2.17 \pm 0.99 *$ & $3.57 \pm 1.04$ & -1.39 & $-2.14--0.64$ & -1.37; Large \\
\hline & & MTV & $2.33 \pm 0.69$ & $3.17 \pm 0.71^{*}$ & -0.84 & $-1.35--0.31$ & -1.19: Moderate \\
\hline & & TMT-1 & $17.09 \pm 1.72^{*}$ & $12.51 \pm 4.81$ & 4,58 & $1.41-4.74$ & 1.27; Large \\
\hline & & TMT-2 & $21.04 \pm 3.49 *$ & $14.39 \pm 3.34$ & 6.65 & $4.11-9.18$ & 1.95; Large \\
\hline
\end{tabular}

MF+SSGs = Small-Sided Games with Mental Fatigue; SSGs=Small-Sided Games without Mental Fatigue VAS-A = Visual Analogue Scale-Anxiety; FS = Feeling Scale; FAS: Felt Arousal Scale; MTV: Motivation; TMT: Trail Making Test *Significant difference p<0.05.

Interception, successful tackle, unsuccessful tackle, 1 touch (successful-unsuccessful), 2 touches (successful-unsuccessful), and lost ball are shown in table 3.

Table 3. Technical responses of amateur soccer players

\begin{tabular}{|c|c|c|c|c|c|c|c|}
\hline \multirow{19}{*}{ 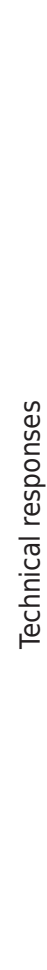 } & \multirow{7}{*}{$\begin{array}{l}\frac{0}{0} \\
\frac{0}{n} \\
\stackrel{1}{0} \\
\stackrel{1}{\sim}\end{array}$} & Variables & $\begin{array}{c}\text { MF+SSGs } \\
\text { Mean } \pm \text { SD }\end{array}$ & $\begin{array}{c}\text { SSGs } \\
\text { Mean } \pm \text { SD }\end{array}$ & $\begin{array}{c}\text { Mean } \\
\text { Difference }\end{array}$ & $\% 95 \mathrm{Cl}$ & $\begin{array}{l}\text { Cohens d } \\
\text { and Rating }\end{array}$ \\
\hline & & Interception & $2.78 \pm 1.17$ & $3.61 \pm 1.04 *$ & 0.83 & $0.21-1.45$ & 0.75; Moderate \\
\hline & & SuccTackle & $0.56 \pm 0.62$ & $1.17 \pm 0.92 *$ & 0.61 & $0.95-1.13$ & 0.78; Moderate \\
\hline & & UnSuccTackle & $1.17 \pm 0.92 *$ & $0.39 \pm 0.50$ & -0.78 & $-1.31-0.25$ & -1.05; Moderate \\
\hline & & 1 Touches (Succ-Unsucc) & $1.22 \pm 0.81$ & $3.06 \pm 1.43 *$ & 1.83 & $0.99-2.67$ & 1.58; Large \\
\hline & & 2 Touches (Succ-Unsucc) & $0.78 \pm 0.55$ & $1.72 \pm 0.83^{*}$ & 0.94 & $0.42-1.47$ & 1.34; Large \\
\hline & & Lost Ball & $4.61 \pm 0.92 *$ & $4.00 \pm 0.97$ & -0.61 & $-1.21--0.02$ & 0.65; Moderate \\
\hline & \multirow{6}{*}{$\begin{array}{l}\frac{d}{0} \\
\frac{0}{n} \\
\frac{1}{0} \\
\dot{m}\end{array}$} & Interception & $3.17 \pm 1.34$ & $3.78 \pm 0.94$ & 0.61 & $-0.15-1.38$ & - \\
\hline & & SuccTackle & $0.67 \pm 0.77$ & $2.39 \pm 0.98 *$ & 1.72 & $1.09-2.36$ & 1.95; Large \\
\hline & & UnSuccTackle & $2.50 \pm 0.79 *$ & $0.67 \pm 0.77$ & -1.83 & $-2.38--1.29$ & -2.50; Very Large \\
\hline & & 1 Touches (Succ-Unsucc) & $3.17 \pm 1.04$ & $4.56 \pm 0.92 *$ & 1.39 & $0.79-1.98$ & 1.42; Large \\
\hline & & 2 Touches (Succ-Unsucc) & $2.17 \pm 0.79$ & $4.11 \pm 0.83^{*}$ & 1.94 & $1.39-2.50$ & 2.39; Very Large \\
\hline & & Lost Ball & $5.89 \pm 1.32 *$ & $3.78 \pm 1.22$ & -2.11 & $-3.09--1.13$ & 1.66; Large \\
\hline & \multirow{6}{*}{$\begin{array}{l}\frac{1}{0} \\
\frac{0}{n} \\
\frac{1}{0} \\
\dot{d} \\
\dot{\gamma}\end{array}$} & Interception & $4.72 \pm 0.96$ & $5.17 \pm 0.92$ & 0.44 & $-0.22-1.11$ & - \\
\hline & & SuccTackle & $1.11 \pm 0.76$ & $1.22 \pm 0.65$ & 0.11 & $-0.43-0.65$ & - \\
\hline & & UnSuccTackle & $1.28 \pm 0.83$ & $1.06 \pm 0.64$ & -0.22 & $-0.75-0.31$ & - \\
\hline & & 1 Touches (Succ-Unsucc) & $4.67 \pm 1.19$ & $6.78 \pm 1.00 *$ & 2.11 & $1.21-1.01$ & 1.92; Large \\
\hline & & 2 Touches (Succ-Unsucc) & $4.67 \pm 0.91$ & $4.78 \pm 0.88$ & 0.11 & $-0.55-0.77$ & - \\
\hline & & Lost Ball & $5.61 \pm 1.20 *$ & $4.67 \pm 0.91$ & -0.94 & $-1.79--0.10$ & -0.88 ; Moderate \\
\hline
\end{tabular}

MF+SSGs=Small-Sided Games with Mental Fatigue; SSGs=Small-Sided Games without-Mental Fatigue SuccTackle: Successful Tackle; UnSuccTackle: Unsuccessful Tackle; Succshot: Successful Shot; 1 Touches [Succ-Unsucc]: 1 Touches Successful SSGs; *Significant difference p<0.05 


\section{DISCUSSION}

The purpose of the current study was to compare the effect of MF intervention in different SSGs. We hypothesized that MF negatively affected soccer players' psychophysiological responses, cognitive performance and technical actions. Study findings showed that MF+SSGs sessions induced higher psychophysiological (VAS-A, FS, FAS, MTV), cognitive demands (TMT), and decreased technical skills compared to SSGs.

The psychophysiological findings in the present study were assessed with VAS-A, FS, FAS, MTV and these scales are frequently used to measure psychophysiological responses in soccer [8, 31]. Previous studies [16, 32] demonstrated that psychophysiological responses in soccer were negatively affected by a MF intervention. Regarding our study psychophysiological results, MF had a detrimental effect on VAS-A, FS, FAS, MTV. Mood [31, 33] and feelings responses [34] changed with MF, higher exhaustion was induced and performance was worse during sport and exercise.

In the current study, MTV reduced in MF+SSGs 2v2 and MF+SSGs 3v3 sessions. In contrast to our results, numerous MF and SSG studies [8, 16, 32] showed no alteration in MTV during MF conditions. Physical responses with perceived mental effort indicated feelings/emotions as a decreasing motivation, recovery demand $[35,36]$ and lead to physical weakness due to lack of energy and unwillingness [18]. Van der Linden et al. [37] demonstrated that MF induces a deteriorating alteration in psychophysiological condition with maintaining performance. Many researchers [38, 39] have stated that an activated mental inhibitor system with increasing mental effort, might cause MF to be a barrier to driving and decreasing willingness. Therefore, individual mood has negatively changed, this and might lead to a lack of energy and unwillingness during physical performance.

Our study provided considerable results concerning the detrimental effects of MF on soccer cognitive performance. TMT was decreased in all MF+SSGs compared to SSGs sessions. There are limited SSGs studies in the literature that supported our study findings [40, 41]. MF also induced deterioration in cognitive performance during SSGs.In the soccer context recent evidence has shown that [16, 17, 42] MF has a negative impact on decision-making performances, including mental-temporal demands, accuracy and response time. The mental performance process with an increase or sustain depends on mental facilitation. Therefore, if the inhibitory system is activated during the cognitive task, it causes a considerable decrease in cognitive performance. However, during a cognitive task, when the inhibitory system is activated, it causes the cognitive performance to decrease [43]. Despite MF having a negative reflection on players' performance, it might be possible to manipulate an increase in the MF effect with environmental conditions, including team cohesion, encouragement, changing the game strategy. Thus, the distraction of players, the unfavourable influence of MF could disappear in the decision-making process.

Essentially, the decision-making process is formed by a combination of perception, attention and working memory [44]. Considering technical actions, MF has reduced technical abilities consisting of interception, successful or unsuccessful tackle, 1 touch (success-unsuccess), 2 touches (success-unsuccess) and lost ball. Our study showed that successful performancerelated technical indicators, such as interception, successful tackle, 1 touch and 2 touches, were decreased in MF+SSGs sessions contrary to increasing unsuccessful tackle and lost ball. Regarding technical performance, previous studies have shown similar results [23, $40,45,46]$. All these results clarify that MF impaired technical skills. In the light of these results, the researchers and coaches might focus on the decision-making process in the relationship between perceived exertion and cognitive system activation.

This study focused on psychophysiological responses, cognitive performance and technical skills of soccer SSGs. We had some restrictions, namely an acute kinematic profile (total 
distance, accelerations and decelerations) and metabolic responses (lactate, cortisol, heart rate) in SSGs. Another limitation of this study was that the sample group were members of an amateur club.

\section{CONCLUSIONS}

The present study indicates the effect of MF on psychophysiological responses, cognitive performance and technical skills. Results demonstrated that before SSGs sessions, MF interventions negatively affected soccer performance including VAS-A, FS, FAS, MTV and TMT. MF interventions could increase perceived effort and lead to deteriorating psychophysiological responses and cognitive function, hence decreasing soccer performance. In the light of these results, it is important for coaches to control the negative effects of MF on players' effort and the potential links with motivation while performing tasks.

\section{REFERENCES}

[1] Lovell G, Townrow J, Thatcher R. Mood states of soccer players in the English leagues: Reflections of an increasing workload. Biol Sport. 2010 Jun 30;27(2):83-8. https://doi.org/10.5604/20831862.913072

[2] Roca A, Ford PR, McRobert AP, Williams AM. Perceptual-cognitive skills and their interaction as a function of task constraints in soccer. J Sport Exerc Psychol. 2013;35(2):144-55. https://doi.org/10.1123/jsep.35.2.144

[3] Sarmento H, Clemente FM, Harper LD, Costa IT da, Owen A, Figueiredo AJ. Small sided games in soccer - A systematic review. Int J Perform Anal Sport. 2018 Sep 3;18(5):693-749. https://doi.org/10.1080/24748668.

[4] Arslan E, Orer G, Clemente F. Running-based high-intensity interval training vs. small-sided game training programs: Effects on the physical performance, psychophysiological responses and technical skills in young soccer players. Biol Sport. 2020;37(2):165-73. https://doi.org/10.5114/biolsport.2020.94237

[5] Arslan E, Soylu Y, Clemente FM, Hazir T, Kin-Isler A, Kilit B. Short-term effects of on-field combined core strength and small-sided games training on physical performance in young soccer players. Biol Sport. 2021;38(4):609-616. https://doi.org/10.5114/biolsport.2021.102865

[6] Sahli H, Selmi O, Zghibi M, et al. Effect of the Verbal Encouragement on Psychophysiological and Affective Responses during Small-Sided Games. Int J Environ Res Public Health. 2020 Nov 29;17(23):8884. https://doi.org/10.3390/ ijerph17238884

[7] Kunrath CA, Nakamura FY, Roca A, Tessitore A, Teoldo Da Costa I. How does mental fatigue affect soccer performance during small-sided games? A cognitive, tactical and physical approach. J Sports Sci. 2020 Aug 2;38(15):1818-28. https://doi.org/10.1080/02640414.2020.1756681

[8] Smith MR, Coutts AJ, Merlini M, Deprez D, Lenoir M, Marcora SM. Mental fatigue impairs soccer-specific physical and technical performance. Med Sci Sport Exerc. 2016;48(2):267-76. https://doi.org/10.1249/MSS.0000000000000762

[9] Clemente F, Sarmento H. Combining small-sided soccer games and running-based methods: A systematic review. Biol Sport. 2021;617-27. https://doi.org/10.5114/biolsport.2021.102932

[10] Clemente FM, Wong DP, Martins FML, Mendes RS. Acute effects of the number of players and scoring method on physiological, physical, and technical performance in small-sided soccer games. Res Sport Med. 2014;22(4):380-97. https://doi.org/10.1080/15438627.2014.951761

[11] Aguiar M, Botelho G, Lago C, Maças V, Sampaio J. A review on the effects of soccer small-sided games. J Hum Kinet. 2012;33:103-13. https://doi.org/10.2478/v10078-012-0049-x

[12] Olthof SBH, Frencken WGP, Lemmink KAPM. The older, the wider: On-field tactical behavior of elite-standard youth soccer players in small-sided games. Hum Mov Sci. 2015;41:92-102. https://doi.org/10.1016/j.humov.2015.02.004

[13] Arslan E, Alemdaroglu U, Koklu Y, Hazir T, Muniroglu S, Karakoc B. Effects of passive and active rest on physiological responses and time motion characteristics in different small sided soccer games. J Hum Kinet. 2017;60(1):123-32. https://doi.org/10.1515/hukin-2017-0095

[14] Köklü Y, Alemdaroğlu U, Cihan H, Wong DP. Effects of bout duration on players' internal and external loads during small-sided games in young soccer players. Int J Sports Physiol Perform. 2017;12(10):1370-7. https://doi.org/10.1123/ ijspp.2016-0584

[15] Clemente FM, Ramirez-Campillo R, Castillo D, et al. Effects of mental fatigue in total running distance and tactical behavior during small-sided games: A systematic review with a meta-analysis in youth and young adult's soccer players. Front Psychol. 2021;12:0-2. https://doi.org/10.3389/fpsyg.2021.656445

[16] Smith MR, Zeuwts L, Lenoir M, Hens N, De Jong LMS, Coutts AJ. Mental fatigue impairs soccer-specific decisionmaking skill. J Sports Sci. 2016;34(14):1297-304. http://dx.doi.org/10.1080/02640414.2016.1156241

[17] Auer S, Kubowitsch S, Süß F, Renkawitz T, Krutsch W, Dendorfer S. Mental stress reduces performance and changes musculoskeletal loading in football-related movements. Sci Med Footb. 2020;:24733938.2020.1860253. https://doi. org/10.1080/24733938.2020.1860253

[18] Marcora SM, Staiano W, Manning V. Mental fatigue impairs physical performance in humans. J Appl Physiol 2009;106(3):857-64. https://doi.org/10.1152/japplphysiol.91324.2008

[19] Boksem MAS, Meijman TF, Lorist MM. Mental fatigue, motivation and action monitoring. Biol Psychol. 2006;72(2):123-32. https://doi.org/10.1016/j.biopsycho.2005.08.007 
[20] van der Linden D, Massar SAA, Schellekens AFA, Ellenbroek BA, Verkes R-J. Disrupted sensorimotor gating due to mental fatigue: Preliminary evidence. Int J Psychophysiol. 2006;62(1):168-74. https://doi.org/10.1016/j. ijpsycho.2006.04.001

[21] Boksem MAS, Meijman TF, Lorist MM. Effects of mental fatigue on attention: An ERP study. Cogn Brain Res. 2005;25(1):107-16. https://doi.org/10.1016/j.cogbrainres.2005.04.011

[22] Lorist MM, Boksem MAS, Ridderinkhof KR. Impaired cognitive control and reduced cingulate activity during mental fatigue. Cogn Brain Res. 2005;24(2):199-205. https://doi.org/10.1016/j.cogbrainres.2005.01.018

[23] Badin OO, Smith MR, Conte D, Coutts AJ. Mental fatigue: impairment of technical performance in small-sided soccer games. Int J Sports Physiol Perform. 2016;11(8):1100-5. https://doi.org/10.1123/ijspp.2015-0710

[24] Hardy CJ, Rejeski WJ. Not what, but how one feels: The measurement of affect during exercise. J Sport Exerc Psychol. 1989;11(3):304-17. https://doi.org/10.1123/jsep.11.3.304

[25] Svebak S, Murgatroyd S. Metamotivational dominance: A multimethod validation of reversal theory constructs. J Pers Soc Psychol. 1985;48(1):107-16. https://doi.org/10.1037/0022-3514.48.1.107

[26] Facco E, Zanette G, Favero L, et al. Toward the validation of Visual Analogue Scale for Anxiety. Anesth Prog. 2011;58(1):8-13. https://doi.org/10.2344/0003-3006-58.1.8

[27] Martin K, Staiano W, Menaspà P, et al. Superior inhibitory control and resistance to mental fatigue in professional road cyclists. Piacentini MF, editor. PLoS One. 2016;11(7):e0159907. https://dx.plos.org/10.1371/journal.pone.0159907

[28] Lezak MD, Howieson DB, Bigler ED, Tranel D. Neuropsychological assessment. 5th Ed. New York: Oxford University Press, New York; 2012.

[29] Van Cutsem J, Marcora S, De Pauw K, Bailey S, Meeusen R, Roelands B. The effects of mental fatigue on physical performance: A systematic review. Sport Med. 2017;47(8):1569-88. https://doi.org/10.1007/s40279-016-0672-0

[30] Hopkins WG, Marshall SW, Batterham AM, Hanin J. Progressive statistics for studies in sports medicine and exercise science. Med Sci Sport Exerc. 2009;41(1):3-13. https://doi.org/10.1249/MSS.0b013e31818cb278

[31] Filipas L, Mottola F, Tagliabue G, La Torre A. The effect of mentally demanding cognitive tasks on rowing performance in young athletes. Psychol Sport Exerc. 2018;52-62. https://doi.org/10.1016/j.psychsport.2018.08.002

[32] Smith MR, Fransen J, Deprez D, Lenoir M, Coutts AJ. Impact of mental fatigue on speed and accuracy components of soccer-specific skills. Sci Med Footb. 2017;1(1):48-52. https://doi.org/10.1080/02640414.2016.1252850

[33] Pires FO, Silva-Júnior FL, Brietzke C, et al. Mental Fatigue alters cortical activation and psychological responses, impairing performance in a distance-based cycling trial. Front Physiol. 2018;9:227. http://journal.frontiersin.org/ article/10.3389/fphys.2018.00227/full

[34] Vrijkotte S, Meeusen R, Vandervaeren C, et al. Mental fatigue and physical and cognitive performance during a 2-bout exercise test. Int J Sports Physiol Perform. 2018;13(4):510-6. https://doi.org/10.3389/fphys.2018.00227

[35] Ishii A, Tanaka M, Watanabe Y. The neural substrates of self-evaluation of mental fatigue: a magnetoencephalography study. Ahveninen J, editor. PLoS One. 2014;9(4):e95763. https://dx.plos.org/10.1371/journal.pone.0095763

[36] Ishii A, Tanaka M, Watanabe Y. Neural mechanisms of mental fatigue. Rev Neurosci. 2014;25(4):469-79. https://doi. org/10.1515/revneuro-2014-0028

[37] van der Linden D, Frese M, Meijman TF. Mental fatigue and the control of cognitive processes: effects on perseveration and planning. Acta Psychol [Amst]. 2003;113(1):45-65. https://doi.org/10.1016/s0001-6918(02)00150-6

[38] Schiphof-Godart L, Roelands B, Hettinga FJ. Drive in sports: How mental fatigue affects endurance performance. Front Psychol. 2018;17:9:1-7. https://doi.org/10.3389/fpsyg.2018.01383

[39] Martin K, Meeusen R, Thompson KG, Keegan R, Rattray B. Mental fatigue impairs endurance performance: A physiological explanation. Sport Med. 2018;48(9):2041-51. https://doi.org/10.1007/s40279-018-0946-9

[40] Trecroci A, Boccolini G, Duca M, Formenti D, Alberti G. Mental fatigue impairs physical activity, technical and decisionmaking performance during small-sided games. Marocolo M, editor. PLoS One. 2020;15(9):e0238461. http://dx.doi. org/10.1371/journal.pone.0238461

[41] Kunrath CA, Nakamura FY, Roca A, Tessitore A, Teoldo Da Costa I. How does mental fatigue affect soccer performance during small-sided games? A cognitive, tactical and physical approach. J Sports Sci. 2020;38(15):1818-28. https:// www.tandfonline.com/doi/full/10.1080/02640414.2020.1756681

[42] Filipas L, Borghi S, La Torre A, Smith MR. Effects of mental fatigue on soccer-specific performance in young players. Sci Med Footb. 2020;1-8. https://doi.org/10.1080/24733938.2020.1823012

[43] Tanaka M, Ishii A, Watanabe Y. Neural effect of mental fatigue on physical fatigue: A magnetoencephalography study. Brain Res. 2014;1542:49-55. https://doi.org/10.1016/j.brainres.2013.10.018

[44] Fortes LS, Nascimento-Júnior JRA, Mortatti AL, Lima-Júnior DRAA de, Ferreira MEC. Effect of dehydration on passing decision making in soccer athletes. res q exerc Sport. 2018;89(3):332-9. https://doi.org/10.1080/0270136 7.2018.1488026

[45] Coutinho D, Gonçalves B, Travassos B, Wong DP, Coutts AJ, Sampaio JE. Mental fatigue and spatial references impair soccer players' physical and tactical performances. Front Psychol. 2017;8. https://doi.org/10.3389/fpsyg.2017.01645

[46] Coutinho D, Gonçalves B, Wong DP, Travassos B, Coutts AJ, Sampaio J. Exploring the effects of mental and muscular fatigue in soccer players' performance. Hum Mov Sci. 2018;58:287-96. https://doi.org/10.1016/j.humov.2018.03.004 